DOI: $10.526 /$ cab.v14i3.15414

\title{
MONITORAMENTO DE FUNGOS TOXIGÊNICOS E AFLATOXINAS EM RAÇÕES UTILIZADAS EM PISCICULTURA
}

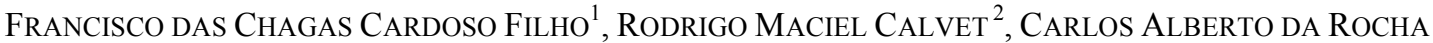 \\ Rosa $^{3}$, Maria Marlúcia Gomes Pereira ${ }^{4}$, Amilton Paulo Raposo Costa ${ }^{4}$, Maria Christina \\ SANCHES MURATORI ${ }^{4}$
}

\footnotetext{
${ }^{1}$ Pós-graduando da Universidade Federal do Piauí, Teresina, PI, Brasil. veterinario_filho@hotmail.com ${ }^{2}$ Professor do Instituto Federal de Educação Ciência e Tecnologia do Maranhão, Caxias, MA, Brasil.

${ }^{3}$ Professor Doutor da Universidade Federal Rural do Rio de Janeiro, Seropédica, RJ, Brasil.

${ }^{4}$ Professores Doutores da Universidade Federal do Piauí, Teresina, PI, Brasil.
}

\begin{abstract}
Objetivou-se determinar a ocorrência de fungos e aflatoxinas em rações para peixes. Foram analisadas 36 amostras de ração para peixes, sendo essas com duas composições proteicas (juvenil/engorda) e em duas formas de uso (lacrado/aberto). Foi realizada a contagem, isolamento e a identificação das espécies de Aspergillus e Penicillium, a capacidade toxígena das cepas da seção Flavi, e ainda fez-se a pesquisa de aflatoxinas na ração. As médias das contagens fúngicas variaram de 2,96 a 4,00
\end{abstract}

UFC/g em $\log _{10}$, e não houve diferença significativas entre os tratamentos. As espécies mais isoladas foram: Aspergillus flavus, Eurotion spp. e Penicillium implicatum. Concluiu-se que as rações analisadas apresentaram elevadas contagens fúngicas, as cepas de Aspergillus flavus isoladas não eram produtoras de aflatoxinas e não foram detectadas aflatoxinas nas amostras de ração analisadas.

PALAVRAS-CHAVE: Alimento animal; Aspergillus; Micobiota; Micotoxinas; Peixes.

\section{MONITORING OF TOXIGENIC FUNGI AND AFLATOXINS IN RATIONS USED IN AQUACULTURE}

\section{ABSTRACT}

The aim of this study was to determine the occurrence of fungi and aflatoxins in fish feeds. We analyzed 36 samples of feed for fish, with two protein compositions (juvenile/fattening) and two forms of use (sealed/open). Aspergillus and Penicillium species were counted, isolated and identified, the toxic capacity of Flavi strains was measured and aflatoxins in the feed were researched. The mean fungal counts ranged from 2.96 to $4.00 \log _{10} \mathrm{CFU} / \mathrm{g}$ and there was no significant difference between treatments. The most isolated species were Aspergillus flavus, Eurotion spp. and Penicillium implicatum. We concluded that the feeds studied had high fungal counts; the isolated Aspergillus flavus strains were not producers of aflatoxin; and aflatoxin was not detected in the feed samples analyzed.

KEYWORDS: Animal feed; Aspergillus; fish; mycobiota; mycotoxins. 


\section{INTRODUÇÃO}

A qualidade dos alimentos destinados à piscicultura é essencial para a produção (NAYLOR et al., 2000). Os piscicultores dispõem de rações comerciais, formuladas especialmente para atender às necessidades nutricionais dos peixes nas diversas fases de crescimento, nas quais são utilizados diversos ingredientes, representando um dos principais custos operacionais na produção. Além disso, as rações contêm os substratos ideais para o crescimento dos fungos e a produção de seus metabólitos, caso haja condições extrínsecas e intrínsecas favoráveis durante seu armazenamento (DANTIGNY et al., 2005).

Os fungos têm sido evidenciados como microrganismos de grande importância para a indústria de alimentos, pois a contaminação por fungos pode causar inúmeras perdas econômicas associadas à redução de nutrientes, da palatabilidade e à presença de micotoxinas, que são metabólitos secundários com potencial para produzir toxicoses no homem e nos animais (SCUSSEL, 2002; PEREIRA et al., 2005).

A presença de fungos na ração não significa necessariamente a presença de micotoxinas (PEREIRA et al., 2002); entretanto, elevadas contagens fúngicas são consideradas um indicativo da presença de micotoxinas no alimento (FAO, 2004). Dentre as micotoxinas, as aflatoxinas, essencialmente produzidas por fungos das espécies Aspergillus flavus e A. Parasiticus, são as mais estudadas.

No Brasil, a ocorrência de aflatoxinas em produtos destinados à alimentação animal tem sido registrada em várias regiões (PEREIRA et al., 2005; ROSA et al., 2006; KELLER et al., 2007; SIMAS et al., 2007). As aflatoxinas são altamente cancerígenas e podem causar toxicidade aguda quando em concentrações elevadas (KABAK \& DOBSON, 2006; KHANAFARI et al., 2007).
Além disso, produzem efeitos mutagênicos, teratogênicos e imunossupressores (MURTHY et al., 2005); tornam-se, portanto, um motivo de preocupação devido aos seus efeitos nocivos sobre a saúde dos seres humanos e animais, além de causarem prejuízos na cadeia produtiva. Diante do exposto torna-se necessário o controle da qualidade dos alimentos fornecidos aos animais por meio da pesquisa de fungos e aflatoxinas.

Deste modo, o objetivo deste trabalho foi determinar a ocorrência de fungos e aflatoxinas em rações comerciais para peixes de fazendas piscicultoras localizadas na cidade de Teresina, Piauí.

\section{MATERIAL E MÉTODOS}

A pesquisa foi realizada na cidade de Teresina, Piauí. Realizou-se um levantamento do número de propriedades piscicultoras da região e constatou-se a existência de 16 no total, das quais três foram sorteadas para a coleta das amostras. Cada fazenda utilizava dois tipos de rações, uma para a fase juvenil e outra para a fase de crescimento. Para amostragem, foram coletados $500 \mathrm{~g}$ de ração que foi retirada de diferentes partes do saco de ração que continha $50 \mathrm{~kg}$, que foram homogeneizados na embalagem original com movimentos giratórios feitos no momento da coleta. Todas as propriedades utilizavam a mesma marca comercial de ração e não utilizavam antifúngicos. Foram coletadas um total de 36 amostras, 12 amostras por propriedade, o correspondente, a seis coletadas por fase (duas) e, de cada fase, três coletas para embalagem lacrada e três para embalagem em uso. As rações ficavam armazenadas dentro de galpões com temperatura média de $32,1^{\circ} \mathrm{C}$, sem contato com a luz solar em um ambiente com umidade relativa média de 62,3 $\%$, como apresentado na Tabela 1.

Tabela 1. Média da umidade relativa (UR) e temperatura dos locais em que eram armazenadas as rações para peixes

\begin{tabular}{lcccc}
\hline & P 1 & P2 & P3 & Média \\
\hline Umidade $(\%)$ & 60,3 & 60,9 & 65,7 & 62,3 \\
Temperatura $\left({ }^{\circ} \mathrm{C}\right)$ & 31,8 & 31,6 & 32,9 & 32,1 \\
\hline
\end{tabular}

$\mathrm{P}=$ propriedades

Após a coleta, as amostras foram levadas para o Laboratório de Controle Microbiológico de 
Alimentos, do Núcleo de Estudos e Pesquisas de Processamento em Alimentos (NUEPPA), da Universidade Federal do Piauí - UFPI para a imediata realização da contagem e identificação dos fungos filamentosos. Após essa etapa, parte da amostra (em torno de $200 \mathrm{~g}$ ) foi levada para a estufa a $70^{\circ} \mathrm{C}$, para eliminação dos fungos e posterior análise de micotoxinas no laboratório de Micologia da Universidad Nacional de Río Cuarto, Córdoba, Argentina.

O procedimento para cada amostra foi iniciado com a trituração em liquidificador, em seguida foi retirada uma alíquota de $25 \mathrm{~g}$, que foi diluída em $225 \mathrm{~mL}$ de água peptonada a $0,1 \%$, formando diluição inicial $\left(10^{-1}\right)$ e, a partir dessa diluição, foram preparadas diluições decimais seriadas até $10^{-3}$. A inoculação foi feita em duplicata, colocando-se uma alíquota de $0,1 \mathrm{~mL}$ por placa de Petri, na superfície do meio de cultivo Dichloran Rose Bengal Cloranfenicol (DRBC), para cada uma das diluições (PITT \& HOCKING, 2009). As placas de DRBC foram incubadas a $25^{\circ} \mathrm{C}$ por sete dias, em ausência de luz. As contagens fúngicas foram realizadas nas placas que apresentaram de 10 a 100 UFC/g (DALCERO et al., 1997; DALCERO et al., 1998). Após contagem de unidades formadoras de colônia (UFC/g), as colônias fúngicas selecionadas para identificação (Aspergillus e Penicillium) foram isoladas e repicadas em tubos contendo agar extrato de malte (MEA) e a identificação das espécies foi realizada de acordo com a chave taxonômica de KLICH (2002) e PITT (1988), respectivamente.

As cepas de Aspergillus flavus isoladas foram cultivadas em placas MEA a $25{ }^{\circ} \mathrm{C}$ por sete dias e testadas quanto à produção de aflatoxinas,. $\mathrm{O}$ micélio foi transferido para um tubo Eppendorf juntamente com $1000 \mu \mathrm{L}$ de clorofórmio. A mistura foi agitada a $4000 \mathrm{rpm}$ por 20 minutos em temperatura ambiente, o micélio foi removido e o extrato de clorofórmio evaporado sob fluxo de $\mathrm{N}_{2}$. O resíduo foi redissolvido em $200 \mu \mathrm{L}$ de clorofórmio. Os extratos foram analisados por cromatografia em camada delgada (CCD) de acordo com SOARES e RODRIGUEZ-AMAYA (1989), em sílica gel 60 F254, TLC chapas de alumínio $(20 \times 20 \mathrm{~cm}$, espessura, $250 \mu \mathrm{m}$, Merck, Alemanha). O liquido carreador foi o tolueno, acetato de etilo e ácido fórmico (50:40:10). O limite de detecção do método utilizado é de $5 \mathrm{mg} / \mathrm{g}$.

Para extração de aflatoxinas na ração, utilizaram-se $25 \mathrm{~g}$ de cada amostra, que foi acrescida em $100 \mathrm{~mL}$ de acetonitrila:água $(84: 16, \mathrm{v} / \mathrm{v})$, e mantida em homogeneização em shaker por 30 minutos. Após a homogeneização, a mistura foi filtrada em papel Whatman $\mathrm{n}^{\circ} 4$ (Whatman, Inc., Clifton, New Jersey, E.U.A.). Retirou-se do filtrado uma alíquota de 5,0 $\mathrm{mL}$ que foi transferida para um tubo de $10 \mathrm{~mL}$. Purificou-se o filtrado com o uso de colunas Multifuncional Mycosep 224 (MFC, Romer Labs ${ }^{\circledR}$, Inc., MO., E.U.A). Após essa limpeza, foram coletados 2,0 mL do extrato purificado, que foram evaporados sob fluxo de $\mathrm{N}_{2}$. No momento da utilização, os extratos foram re-dissolvidos utilizando $200 \mu \mathrm{L}$ da fase móvel. A detecção de aflatoxinas e quantificação de cada amostra foi realizada por cromatografia líquida de alta eficiência (CLAE) de acordo com TRUCKESS et al. (1994). Uma alíquota $(200 \mu \mathrm{L})$ foi derivatizada com $700 \mu \mathrm{L}$ de ácido acético-água-ácido trifluoroacético $(20: 10: 70, v / v)$. Separações cromatográficas foram realizadas em uma coluna de fase reversa (Silica Gel, $150 \times 4,6 \mathrm{~mm}$ id, tamanho de partículas de $5 \mu \mathrm{m}$, Varian, Inc. Palo Alto, E.U.A.). A fase móvel empregada foi o acetonitrila:metanol:água $(1: 1: 4 \mathrm{v} /$ $\mathrm{v} / \mathrm{v})$, o volume injetado foi de $20 \mu \mathrm{L}$ e a velocidade do fluxo foi de $1,5 \mathrm{~mL}$ por minuto. A detecção das aflatoxinas foi por fluorescência utilizando um comprimento de onda de $360 \mathrm{~nm}$ de excitação e 440 $\mathrm{nm}$ de emissão. As curvas padrão foram construídas com diferentes níveis de aflatoxinas. O limite de detecção do método foi de $0,4 \mathrm{ng} / \mathrm{mL}$.

Foi realizado um delineamento fatorial $2 \times 2$ (tipos de ração - juvenil e crescimento - e forma de armazenamento - lacrada e aberta), e os resultados das contagens foram transformados em $\log _{10}$, correlacionados e análise de variância foi realizada com significância $(p<0,05)$, utilizando o programa estatístico SIGMA STAT (1994).

\section{RESULTADOS E DISCUSSÃO}

Na Tabela 2 encontram-se os resultados das contagens de fungos filamentosos e leveduras isolados de dois tipos de rações para peixes, segundo as formas de armazenamento.

Não houve diferença estatisticamente significativa entre as médias dos tratamentos pesquisados, ou seja, as médias de contagem de fungos foram estatisticamente semelhantes nas rações.

No Brasil não há padrões legais para contagem de fungos em ração animal, mas, segundo o padrão utilizado em outros países para certificação de ração animal (GMP, 2008), recomendam-se contagens não superiores a $4,00 \mathrm{UFC} / \mathrm{g}$ em $\log _{10}$. Com base nesse padrão, quando considerado o valor de cada amostra, observou-se que 12 (33\%) das 36 amostras, apresentaram valores que excedem esses limites, considerados como padrão que visa garantir a qualidade higiênica do produto.

Esses resultados sugerem uma alta atividade 
de fungos que podem afetar a palatabilidade da ração e reduzir a absorção de nutrientes pelos animais, determinando um substrato de baixa qualidade e diminuição da produção.

Os fungos isolados também podem produzir micotoxinas, caso encontrem condições favoráveis para sua multiplicação (DANTIGNY et al., 2005); portanto, cuidados nas etapas de processamento da ração são necessários, pois esses resultados indicam falhas durante o seu processamento e armazenamento (FAO, 2004).

A micobiota total (Tabela 2) foi semelhante aos valores relatados por outros autores sobre cereais e rações (ACCENSI et al., 2004; ROSA et al., 2006; KELLER et al., 2007) e superior aos estudos realizados por OLIVEIRA et al.(2007) e RIBEIRO et al. (2009). Esse nível de contaminação pode variar em função das condições climáticas da região, do período de coleta, das condições de processamento do alimento e da forma em que está armazenado (RIBEIRO et al., 2009).

$\mathrm{Na}$ Tabela 3, observa-se a ocorrência de fungos filamentosos isolados da ração para peixes. Pode-se constatar que o gênero de maior ocorrência foi o Aspergillus e Teleomorfos com mais de 50\% das cepas isoladas, seguidos de Penicillium, Cladosporium e Fusarium. Alguns autores relataram resultados semelhantes (MAGNOLI et al., 2002; KELLER et al., 2007).

De acordo com RIBEIRO et al. (2003), o gênero Aspergillus é considerado como o principal deteriorador de sementes e grãos, causando danos, descoloração e alterações nutricionais.

Tabela 2. Médias das contagens de fungos filamentosos e leveduras por propriedade isoladas de dois tipos de rações para peixes

\begin{tabular}{lcccccc}
\hline $\begin{array}{l}\text { Tipos/ } \\
\text { condições }\end{array}$ & $\begin{array}{c}\text { Médias } \\
\text { P 1 }\end{array}$ & $\begin{array}{c}\text { Médias } \\
\text { P 2 }\end{array}$ & $\begin{array}{c}\text { Médias } \\
\text { P 3 }\end{array}$ & $\begin{array}{c}\text { Médias das } \\
\text { Contagens } \\
\text { fúngicas }\end{array}$ & Variação & $\begin{array}{c}(\%)>\text { acima do } \\
\text { limite indicado(*) }\end{array}$ \\
\hline J/L $(n=9)$ & 3,08 & 4,74 & 2,00 & 3,27 & $1,70-4,83$ & $44,4(\mathrm{n}=4)$ \\
J/A $(\mathrm{n}=9)$ & 3,80 & 4,88 & 3,33 & 4,00 & $2,20-5,00$ & $66,6(\mathrm{n}=6)$ \\
C/L $(\mathrm{n}=9)$ & 3,48 & 2,53 & 2,88 & 2,96 & $1,70-3,87$ & $0(\mathrm{n}=0)$ \\
C/A $(\mathrm{n}=9)$ & 3,21 & 3,21 & 2,96 & 3,07 & $1,70-4,59$ & $22,2(\mathrm{n}=2)$ \\
\hline
\end{tabular}

$\mathrm{UFC} / \mathrm{g}=$ unidade formadora de colônias por grama em $\log _{10}$. Nível máximo recomendável: 4,00 $\log _{10}$ UFC/g pela Good Manufacturing Practices - GMP $(2008) ;(*):(\mathrm{P}=0.30) . \mathrm{J} / \mathrm{L}=$ juvenil/lacrado; $\mathrm{J} / \mathrm{A}=$ juvenil/aberto; $\mathrm{C} / \mathrm{L}=$ crescimento/lacrado; $\mathrm{C} / \mathrm{A}=$ crescimento/aberto. $\mathrm{N}=$ amostras; $\mathrm{P}=$ propriedades

Tabela 3. Ocorrência (\%) de fungos filamentosos isolados das amostras de ração para peixes

\begin{tabular}{lcc}
\hline Gênero Fúngico & $\mathrm{N}^{\text {o }}$ de Isolados & Ocorrência (\%) \\
\hline Aspergillus e teleomorfos & 41 & 56,16 \\
Penicillium & 14 & 19,18 \\
Cladosporium & 12 & 16,44 \\
Fusarium & 06 & 8,22 \\
\hline Total & 73,0 & 100 \\
\hline
\end{tabular}

Tabela 4. Densidade relativa (\%) de espécies de Aspergillus isoladas das amostras de ração para peixes.

\begin{tabular}{lcc}
\hline Espécies & Total de cepas & Frequência (\%) \\
\hline A. flavus & 26 & 60,47 \\
Eurotium spp. & 08 & 18,61 \\
A. oryzae & 03 & 6,98 \\
A. fumigatus & 03 & 6,98 \\
A. terreus & 01 & 2,32 \\
A. candidus & 01 & 2,32 \\
A. penicillioides & 01 & 2.32 \\
\hline Total & 43 & 100 \\
\hline
\end{tabular}

Dentre as espécies de Aspergillus, podemos observar (Tabela 4) que a espécie que predominou 
com mais de $60 \%$ das cepas foi o A. flavus, concordando com estudos de outros autores (DALCERO et al., 1998; ACCENSI et al., 2004); as demais espécies encontradas foram os seus teleomorfos Eurotium, A. oryzae, A. fumigatus, A. terreus, A. candidus e A. penicillioides. A presença de $A$. flavus nas rações representa um perigo potencial, pois pode ocasionar enfermidades nos trabalhadores que diretamente estão em contato com ela, como a aspergilose (AKAN et al., 2002), alergias e problemas respiratórios pelo contato e inalação de conídios, como também causar problemas na produção animal, por ser um potencial produtor de micotoxinas.

Todas as cepas de $A$. flavus foram analisadas quanto à capacidade de produzirem aflatoxina, porém nenhuma das cepas demonstrou capacidade toxígena pela técnica empregada. Sabe-se, entretanto, que existem outras técnicas que podem ser empregadas para essa detecção como a biologia molecular e a cromatografia líquida de alta eficiência. A importância dessa verificação é que, caso fossem produtoras, seria um grande risco na alimentação dos peixes, pois a aflatoxina B1 nas rações pode acarretar, quando ingerida, quadros de carcinoma hepatocelular, redução do peso corporal, alterações hematológicas e necrose dos hepatócitos (MANNING et al., 2005; WILLIANS et al., 2009).

$\mathrm{Na}$ Tabela 5, podemos observar a densidade relativa das espécies de Penicillium, sendo que apenas quatro espécies deste gênero foram isoladas nesse estudo, diferentemente da variedade de espécies encontradas por outros autores também trabalhando com ração animal (MAGNOLI et al., 2006; ROSA et al., 2006).

Tabela 5. Densidade relativa (\%) de espécies de Penicillium isoladas das amostras de ração para peixes

\begin{tabular}{lll}
\hline Espécies & Total & Freqüência (\%) \\
\hline P.implicatum & 08 & 57,15 \\
P.restrictum & 03 & 21,43 \\
P.citrinum & 02 & 14,28 \\
P.rugulosum & 01 & 7,14 \\
\hline Total & 14 & 100 \\
\hline
\end{tabular}

Muitas das espécies isoladas neste estudo estão associadas com a perda da qualidade da ração e podem ser potenciais produtoras de micotoxinas. $\mathrm{O}$ isolamento dessas espécies a partir de rações animais tem sido realizado por outros pesquisadores (MAGNOLI et al., 2006; ROSA et al., 2006; KELLER et al., 2007).

Foi verificada alta incidência de fungos nas rações para peixes coletadas e avaliadas em Teresina, PI, com quantidade significativa de cepas de Aspergillus flavus (Tab. 4), e a alta contagem fúngica é considerada indicador da provável presença de micotoxinas (FARIAS et al., 2000). No entanto, aflatoxinas não foram detectadas nas amostras analisadas em nosso estudo. Isso pode ser atribuído ao curto espaço de tempo para a formação dessas ou mesmo ao número reduzido de linhagens fúngicas com potencial toxigênico, de acordo com PEREIRA et al. (2002). Em trabalho realizado por GUERRA et al. (2005) com alimentos para equinos, também não foi detectada a presença de aflatoxinas nas 50 amostras. Esse resultado negativo também pode ser decorrente de uma amostragem feita de forma inadequada.

Os níveis de aflatoxinas em rações para peixes utilizadas em Londrina, Paraná, variaram desde não detectável a 15,60 ng/g (HASHIMOTO et al., 2003). Já NUNES (2009), pesquisando aflatoxinas em rações para peixes oriundas da indústria da cidade de Teresina, Piauí, constatou também a presença dessas micotoxinas em várias amostras de ração, como também na matéria prima utilizada para a sua formulação.

Existe uma recomendação do Ministério da Saúde de que, para qualquer matéria prima utilizada diretamente ou como ingrediente nas rações, ou para a própria ração, os valores de aflatoxinas não devem ser superiores a $50 \mu \mathrm{g} / \mathrm{kg}$ (BRASIL, 2002); portanto, todas as amostras utilizadas neste estudo estão de acordo com o padrão no que corresponde à presença de aflatoxinas, pois elas não foram detectadas em nenhuma das 36 amostras.

Portanto, estudos que determinem a micobiota fúngica e suas micotoxinas nos alimentos são importantes porque permitem obter informações inerentes à qualidade do produto e realizar ações preventivas nas fases de produção, colheita e armazenamento dos produtos para que haja o controle dos fungos e seus metabólitos, somadas ao emprego de boas práticas de fabricação a fim de se 
reduzir a contaminação dos alimentos e evitar perdas na produção animal e riscos para a saúde de animais e humanos.

\section{CONCLUSÕES}

Concluiu-se que as rações analisadas apresentaram elevadas contagens fúngicas. As cepas de Aspergillus flavus isoladas não eram produtoras de aflatoxinas. Não foram detectadas aflatoxinas nas amostras de ração analisadas.

\section{REFERÊNCIAS}

ACCENSI,F.; ABARCA,M.L.; CABAÑES,F.J. Occurrence of Aspergillus species in mixed feeds and component raw materials and their ability to produce ochratoxin A. Food Microbiology, v. 21, 623-627, 2004.

AKAN, M.; HAZIROGLU, R.; ILHAN, Z.; SAREYYUPOGLU, B.; TUNCA, R. A. Case of 9 aspergillosis in a broiler breeder flock. Avian Diseases, v.42, n.2, p.497-501, april/june, 2002.

BINDER,E.M.; TAN,L..M.; CHIN, L.J.; HANDL,J.; RICHARD,J. Wordwide occurrence of mycotoxins in commodities, feeds and feed ingredients. Animal Feed Science and Technology, 137, 265-282, 2007.

BRASIL. Resolução da Diretoria Colegiada No 274 , da ANVISA, de 15 de outubro de 2002.Disponível em: $<$ http://www.anvisa.gov.br/anvisalegis/resol/2002/274 02rdc.htm > Acessado em: 12 set. 2011

DALCERO,A.;MAGNOLI,C.;

CHIACCHIERA,S.; PALACIOS,G.; REYNOSO,M. Mycroflora and incidence of afaltoxin B1, zearalenone and deoxynivalenol in poultry feeds in Argentina. Mycopathologia, v. 137, n. 3, p 179-184, 1997.

DALCERO,A.; MAGNOLI,C.; LUNA,M.; ANCASI,G.; REYNOSO,M. CHIACCHIERA,S.; MIAZZO,R.; PALACIO,G. Mycoflora and naturally ocurring mycotoxins in poultry feds in Argentina. Mycopathologia, v. 141, n. 1, p 37-43, 1998.

DANTIGNY, P.; GUILMART, A.; BENSOUSSAN, M. Basis of predictive mycology. International Journal Food Microbiology. 100, 187-196, 2005.

FAO. Almacenaje.

2004.Disponível em: $<$ http://www.fao.org> Acessado em: 23 set. 2010

FARIAS, A. X.; ROBBS, C. F.; BITTENCOURT, A.; ANDERSEN, P. M.; CORRÊA, T. B. S. Contaminação endógena por Aspergillus spp. em milho pós-colheita no Estado do Paraná. Pesquisa Agropecuária Brasileira, v.35, n.3, p.617- 621, 2000 .

FONSECA, H (2006). Os fungos e a deterioração de alimentos. Disponível: $<$ http: www.micotoxinas.com.br/boletim4.htm $>$. Acessado em:
27 jun. 2010.

GMP (Good Manufacturing Practices). Certification Scheme Animal Feed. Sector 2008, Appendix 1: Product standards; Regulations on Product Standards in the Animal Feed Sector. GMP14, p. 1- 39. 2008. Disponível em: $\quad<$ http://www.bezpecnakrmiva.cz/soubory/gmp_standard_08_EN.pdf $>$ Acessado em: 23 set. 2010

GUERRA, M. M; MARTINS, H.; GOUVEIA, M. F.; BERNADO, F. Aspectos da seguraça sanitária dos alimentos compostos para cavalos. Revista Portuguesa de Zootecnia, v.12, n. 2, p. 63-75, 2005.

HASHIMOTO,E.H.; SANTOS,M.A.; ONO,E.Y.S.; HAYASHI, C.; BRACARENSE, A. P. F. R. L.; HIROOKA, E.Y. Bromatologia e contaminação com fumonisina e aflatoxina em rações utilizadas na piscicultura da região de Londrina, Estado do Paraná, Brasil. Semina: Ciências Agrárias, v. 24, n. 1, p. 123-132, jan./jun. 2003.

KABAK, B.; DOBSON, A.D. Strategies to prevent mycotoxin contamination of food and animal feed: a review. Critical Reviews in Food Science Nutrition. 46, 593-619, 2006.

KELLER, K.M; QUEIROZ, B. D.; KELLER, L. A. M.; CAVAGLIERI, L. R.; PEREYRA, M. L. G.; DALCERO, A. M.; ROSA, C. A. R. The mycobiota and toxicity of equine feeds. Veterinary Research Communications, v.31, n. 8, p. 1037-1045, 2007.

KHANAFARI, A.; SOUDI, H.; MIRABOULFATHI, M. Biocontrol of Aspergillus flavus and aflatoxin B1 production in corn. Iranian Journal of Environmental Health Science \& Engineering. v. 4, p.163-168, 2007.

$\mathrm{KLICH}, \mathrm{M}$. A. A laboratory guide to the common Aspergillus species and their teleomorphs. CSIRO Division of Food Processing, Australia, 2002. 116p.

MAGNOLI, C.; ASTORECA, A.; PONSONE, L., CHIACCHIERA, S.; DALCERO, A. Ochratoxin A and the occurrence of ochratoxin A- producing black aspergilli in stored peanut seeds from Córdoba, Argentina. Journal of the Science of Food and Agriculture, v. 86, p. 23692373, 2006.

MAGNOLI, C.; CHIACCHIERA, S.M.; MIAZZO, R., PALACIO, G.; ANGELETTI, A.; HALLAK, C.; DALCERO, A. The mycoflora and toxicity of feedstuffs from a production plant in Cordoba, Argentina. Mycotoxin Reserch, v.18, p. 7-22, 2002.

MANNING, B. B.; MENGHE, H. L.; ROBINSON, E. H. Aflatoxins from moldy corn cause no reductions in channel catfish Ictalurus punctutus performance. Journal of the World Aquaculture Society, v. 36, n.1, p. 59-67, 2005 .

MARTINS, H. M.;MARTINS, M. L.Qualidade micológica de rações para bovinos (Portugal:1996-1999). Revista Portuguesa de Ciências Veterinárias, v. 96, n.538 p.85-88, 2001. 
MURTHY, G.S., TOWNSEND, D.E., MEERDINK, G.L., BARGREN,G.L., TUMBLESON, M.E., Singh, V. Effect of aflatoxin $\mathrm{B} 1$ on dry-grind ethanol process. Cereal Chemistry, v.82, p. 302-304,2005.

NAYLOR, R.L.; GOLDBURG, R.J.; PRIMAVERA, J.H.; KAUTSKY, N. BEVERIDGE, M. C. M.; CLAY, J.; FOLKE,L.; LUBCHENCO, J.; MOONEY, H.; YROELL, M. Effect of aquaculture on world fish supplies. Nature, v.405, p.1017-1024, 2000.

NUNES, E.M.C.G. Microbiota fúngica nos ingredientes e em ração para piscicultura. 2009. 58 f. Dissertação (Mestrado em Ciência Animal) - Pós Graduação em Ciência Animal, Universidade Federal do Piaú, Teresina.: $<$ http://

ww.ufpi.br/subsiteFiles/ciencianimal/arquivos/.../Dissert\% 20Etelvina.pdf > Acessado em: 08 jun. 2011

OLIVEIRA, G.R.; RIBEIRO, J.M.M.; FRAGA, M.E.; CAVAGLIERI, L. R.; DIREITO, G. M.; KELLER, K. M.; DALCERO, A. M.; ROSA, C. A. R. Mycobiota in poultry feeds and natural occurrence of aflatoxins, fumonisins and zearalenone in the Rio de Janeiro State, Brazil. Mycopathologia, v.162, p.355-362, 2007.

PEREIRA, M.M.G.; CARVALHO, E.P.; PRADO, G. Crescimento e produção de aflatoxinas por aspergillus flavus e aspergillus parasiticus. Boletim CEPPA, v.20, v.1, p. 141-156, 2002.

PEREIRA, M. M. G.; CARVALHO, E.P.; PRADO, G.; ROSA, C.A.R.; VELOSO, T.; SOUZA, L.A.F.; RIBEIRO, J.M.M. Aflatoxinas em alimentos destinados a bovinos e em amostras de leite da região de Lavras, Minas Gerais - Brasil, Ciência e Agrotecnologia, v. 29, n. 1, p.106-112, 2005 .

PITT, J.I. A Laboratory guide to commom Penicillium species. 2 nd ed. Sydney, Australia: CSIRO, Division of Food Processing. 1988. 186p.

PITT, J. I.; HOCKING, A. D. Fungi and spoliage. 2 ed. London: Blackie academic and Professional, 2009. 593p.

RIBEIRO,J.M.M.; CAVAGLIERI, L.R.; VITAL,H.C.; KRUGER, C. D.; ROSA, C. A. R. Radiação gama sobre a micobiota de ração avícola e Aspergillus spp. Ciência Rural, Santa Maria, Online,v. 39, n. 5, p.1452-1458, 2009.

RIBEIRO, S.A.A.L.; CAVALCANTI, M.A.Q.; FERNANDES, M. J. S.; LIMA, D. M. M. Fungos filamentosos isolados de produtos derivados do milho comercializados em Recife, Pernambuco. Revista Brasileira de Botânica, v. 26, n.2, p. 223-229. 2003.

SOARES, L.M.V.; RODRIGUEZ-AMAYA, D.B.
Pesquisa de aflatoxinas, ocratoxinas A, zearalenona e sterigmatocystin em alguns alimentos brasileiros usando método multitoxina de cromatografia de camada delgada. Journal of Association of Official Analitlical Chemistries, v.72, p. 22-26, 1989

ROSA, C. A.R; RIBEIRO, J.M.M.; FRAGA, M.J.; GATTI, M.; CAVAGLIERI, L.R.; MAGNOLI, C.E.; DALCERO, A.M.; LOPES, C.W.G. Mycoflora of poultry feeds and ochratoxin-producing ability of isolated Aspergillus and Penicillium species. Veterinary Microbiology, v. 113, n.1-2, p.89-96, 2006

ROSSETTO, C. A.; SILVA, O. F.; ARAÚJO, A. E. S. Influência da calagem, da época de colheita e da secagem na incidência de fungos e aflatoxinas em grãos de amendoim armazenados. Ciência Rural, v.35, n.2, p.309315, 2005.

SASSAHARA, M.; YANAKA, E. K.; NETTO, D. P. Ocorrência de aflatoxina e zearalenona em alimentos destinados ao gado leiteiro na região Norte do Estado do Paraná. Semina, v.24, p.63- 72, 2003.

SCUSSEL, V.M. Fungos em grãos armazenados. In: Lorini, I., Miike, LH, Scussel, VM Armazenagem de grãos. IBG (Campinas, São Paulo, Brasil), 675-804, 2002.

SIGMA STAT for windows version 1.0. Jandel Corporation, 1994.

SIMAS, M. M.; BOTURA M. B.; CORREA, B. Determination of fungal microbiota and mycotoxins in brewers grain used in dairy cattle feeding in the State of Bahia, Brazil. Food Control, v.18, p. 404-408, 2007.

TRUCKSESS, M.W.; STACK, M.E.; NESHIM, S.; ALBERT, R. H.; ROMER, T. R. Multifunctional column coupled with liquid chromatography for determination of aflatoxins B1, B2, G1, G2 in corn, almonds, Brazil nuts, peanuts and pistachionuts: collaborative study. Journal of Association of Analytical Communities International, 1994. Int 6, 1512-1521.

WILLIAMS, D. E.; ORNER, G.; WILLARD, K.D.; TILTON, S.; HENDRICKS, J. D.; PEREIRA, C.; BENNINGHOFF, A. D. Rainbow trout (Oncorhynchus mykiss) and ultra-low dose cancer studies. Comparative Biochemistry and Physiology- Part C: Toxicology \& Pharmacology, v.149, n.2, p.175-181, 2009.

YANAKA, E.K., NETTO, D.P.; SASSAHARA, M.; TAJIRI, A.N.; OLIVEIRA, A.M. Avaliação da presença de micotoxinas em milho e rações destinadas à avicultura comercial de postura nas regiões norte e noroeste do Estado do Paraná. Pesquisa Veterinária Brasileira, v.24, supl.1, p.79, 2004.

Protocolado em: 19 ago. 2011. Aceito em 8 maio 2013. 